13

\title{
Исследование ультратонких сверхпроводящих пленок нитрида ниобия, полученных методом атомно-слоевого осаждения
}

\author{
() М.В. Шибалов, Н.В. Порохов, А.М. Мумляков, И.В. Трофимов, Г.Д. Дюдьбин, Е.Р. Тимофеева, \\ А.М. Тагаченков, Ю.В. Ануфриев, Е.В. Зенова, М.А. Тархов
}

Институт нанотехнологий микроэлектроники РАН, 119991 Москва, Россия

e-mail: shibalov.m@inme-ras.ru

Поступило в Редакцию 8 сентября 2020 г.

В окончательной редакции 19 октября 2020 г.

Принято к публикации 6 ноября 2020 г.

\begin{abstract}
Представлен способ осаждения ультратонких сверхпроводящих пленок $\mathrm{NbN}_{x}$ методом атомно-слоевого осаждения, усиленного плазмой из металлорганического прекурсора и газовой смеси $\mathrm{H}_{2} / \mathrm{Ar}$, используемой в качестве реактанта. Полученные образцы характеризовались измерением удельного сопротивления, спектральной эллипсометрией, атомно-силовой микроскопией и измерениями сверхпроводящих характеристик. Определены оптимальные параметры соотношения газов $\mathrm{H}_{2} / \mathrm{Ar}$, при которых удельное сопротивление пленок $\mathrm{NbN}_{x}$ минимально. Проведен сравнительный анализ удельного сопротивления полученных пленок $\mathrm{NbN}_{x}$. Исследована зависимость температуры перехода в сверхпроводящее состояние от толщины пленки. Достигнута температура перехода в $13.7 \mathrm{~K}$ и критическая плотность тока $0.7 \mathrm{MA} / \mathrm{cm}^{2}$. Высокая однородность пленки, прецизионный контроль толщины и температура осаждения $350^{\circ} \mathrm{C}$ дают возможность использовать данные пленки в производстве полевых транзисторов и в функциональных устройствах различного назначения, например, болометрах на горячих электронах, детекторах на кинетической индуктивности и сверхпроводниковых однофотонных детекторах.
\end{abstract}

Ключевые слова: атомно-слоевое осаждение, сверхпроводники, нитрид ниобия, температура перехода, критическая плотность тока.

DOI: 10.21883/JTF.2021.04.50632.262-20

\section{Введение}

Нитрид ниобия ввиду комбинации своих уникальных физических и химических свойств имеет множество различных применений. Высокая температура плавления, химическая и механическая стойкость [1,2], относительно хорошая электрическая проводимость дают возможность использовать этот материал в качестве износостойких и биосовместимых покрытий [3,4], диффузионного барьера для создания медной металлизации [5] и электрода затвора для устройств на КМОП (комплементарная логика с транзисторами на металлоксид-полупроводнике) [6]. Также множество применений этого материала связано с его сверхпроводящими свойствами. Пленки нитрида ниобия используют для создания детекторов [7-10] и микроволновых параметрических усилителей [11].

Пленки нитрида ниобия получают такими методами, как магнетронное распыление [12], импульсное лазерное осаждение [13], металлорганическое осаждение из газовой фазы [14], атомно-слоевое осаждение [15]. Среди перечисленных методов высокий интерес представляет атомно-слоевое осаждение, усиленное плазмой (PEALD). За последнее время большая часть научных работ посвящена созданию ультратонких сверхпроводящих пленок методом PEALD [16,17]. В этом методе используются последовательные самоограниченные и поверхностно-контролируемые газофазные химические реакции, позволяющие управлять составом пленки и прецизионно контролировать скорость осаждения и соответственно толщину. Также преимуществом метода является осаждение ультратонких пленок (толщиной несколько нанометров) при низких температурах осаждения $\left(300-350^{\circ} \mathrm{C}\right)$, что позволяет интегрировать пленки в оптические системы (четвертьволновый резонатор) для повышения эффективности сверхпроводниковых детекторов.

При плазменном атомно-слоевом осаждении нитрида ниобия ввиду сложности химических реакций, происходящих на поверхности, получение пленок с определенными параметрами затруднительно. В настоящей работе экспериментально исследуются удельное сопротивление пленок в зависимости от отношения газовой смеси $\mathrm{H}_{2} / \mathrm{Ar}$, сплошность покрытия, шероховатость, а также некоторые параметры сверхпроводимости, такие как температура перехода в сверхпроводящее состояние и критическая плотность тока.

\section{1. Эксперимент}

Для эксперимента выбраны кремниевые пластины $p$-типа $\langle 100\rangle$ диаметром $100 \mathrm{~mm}$, на которые методом плазмохимического осаждения (PECVD) из смеси $\mathrm{SiH}_{4} / \mathrm{N}_{2} \mathrm{O}$ была осаждена пленка $\mathrm{SiO}_{x}$ при температуре 
$350^{\circ} \mathrm{C}$. После чего пластины, не контактируя с атмосферой, отправлялись в модуль атомно-слоевого осаждения. Пленка нитрида ниобия осаждалась повторением реакционных серий, включающих в себя подачу металлорганического прекурсора TBTDEN (tris(diethylamido)(tertbutylimido) niobium) и водородно-аргонной индуктивносвязанной плазмы (ICP) в качестве реактанта. Длительность импульса подачи металлоорганического прекурсоpa TBTDEN составляла $3 \mathrm{~s}$. Время воздействия плазмы ICP газовой смеси $\mathrm{H}_{2} / \mathrm{Ar}$ составляла $43 \mathrm{~s}$. Осаждение проходило при температуре столика $350^{\circ} \mathrm{C}$ и стенок камеры $130^{\circ} \mathrm{C}$. Для обеспечения необходимого давления паров температура прекурсора TBTDEN составляла $95^{\circ} \mathrm{C}$. Мощность на ICР-источнике составляла $100 \mathrm{~W}$. Давление в камере определялось отношением потоков газов $\mathrm{H}_{2} / \mathrm{Ar}$ при полностью открытой заслонке.

При фиксированном количестве циклов, равном 150 , что соответствовало толщине пленки $\sim 11 \mathrm{~nm}$, исследовалась зависимость удельного сопротивления пленок в зависимости от отношения газовой смеси реактанта $\mathrm{H}_{2} / \mathrm{Ar}$. В работах $[18,19]$ для получения пленок с низким удельным сопротивлением во время воздействия плазмы дополнительно подавали активное смещение на столик, что повышает энергию ионов, бомбардирующих подложку, что приводит к уменьшению удельного сопротивления. Ввиду отсутствия возможности подачи активного смещения и на основании работы [20], в которой сообщается об увеличении потока и энергии ионов при понижении давления в процессной камере, давление при всех отношениях смеси $\mathrm{H}_{2} / \mathrm{Ar}$ находилось на минимально возможном уровне.

Поверхностное сопротивление пленок измерялось четырехзондовым методом на зондовой станции 4D Automatic Four Point Probe Meter Model 280 с возможностью построения карты распределения по поверхности $100 \mathrm{~mm}$ пластины. Удельное сопротивление рассчитано путем произведения среднего значения поверхностного сопротивления пленки и толщины пленки, определенной с помощью спектрального эллипсометра.

Известно, что толщину тонких металлических пленок можно измерять с высокой точностью методами эллипсометрии [21]. Спектр отражения от пленок был обработан с помощью модели, включающей в себя осциллятор Друде (учитывает поглощения в ближнем ИК диапазоне) и осциллятор Лоренца. Для подтверждения правильности методики измерения тонких металлических пленок оптической эллипсометрией проведено измерение толщины пленки методом просвечивающей электронной микроскопии и рентгеновской рефлектометрией. На основании толщины пленок рассчитывалась средняя скорость роста пленки за цикл и удельное сопротивление.

Для исследования морфологии поверхности использовались методы атомно-силовой микроскопии. Морфология поверхности исследовалась на площади $1 \mu \mathrm{m}^{2}$.

Измерение критической температуры перехода в сверхпроводящее состояние и критической плотно- сти тока является неотъемлемой частью исследования сверхпроводящих пленок и структур на их основе [22,23]. Измерение сверхпроводящих свойств проводилось на двух различных криостатах. Измерение критической температуры пленок проводилось бесконтактным методом экранирования магнитного поля. Критическая плотность тока измерялась в криостате замкнутого цикла Гиффорда-МакМагона, охлаждаемого до температуры $2.3 \mathrm{~K}$. Термостабилизация образца осуществлялась с помощью резистивного нагревателя и криогенного термометра, объединенных в систему PID (пропорционально-интегрально-дифференцирующий) регулятора. При стабилизации рабочей температуры измерялась вольт-амперная характеристика в режиме стабилизации напряжения. По величине критического тока и топологии исследуемого образца можно судить о критической плотности тока.

\section{2. Результаты и обсуждение}

Исходя из измеренных толщин пленок и их поверхностном сопротивлении, получена зависимость удельного сопротивления от отношения газовой смеси, изображенная на рис. 1.

При малом отношении $\mathrm{H}_{2} / \mathrm{Ar}$ удельное сопротивление было высоким и составляло $1476 \mu \Omega \cdot \mathrm{cm}$. Мы предполагаем, что такое высокое удельное сопротивление можно объяснить недостатком восстановительного реактанта, т.е. водорода. Вследствие чего количество ниобия, связанного с кислородом из остаточной атмосферы камеры, велико. При повышении отношения газовой смеси происходит частичное восстановление оксида ниобия. Видно, что для выбранных экспериментальных точек кривая имеет экстремум при отношении смеси, равным 0.5 (рис. 1). Удельное сопротивление при этом составляет $260 \mu \Omega \cdot \mathrm{cm}$. Скорость роста за цикл при этом составила $0.072 \mathrm{~nm} /$ cycle. Дальнейшее увеличение отношения

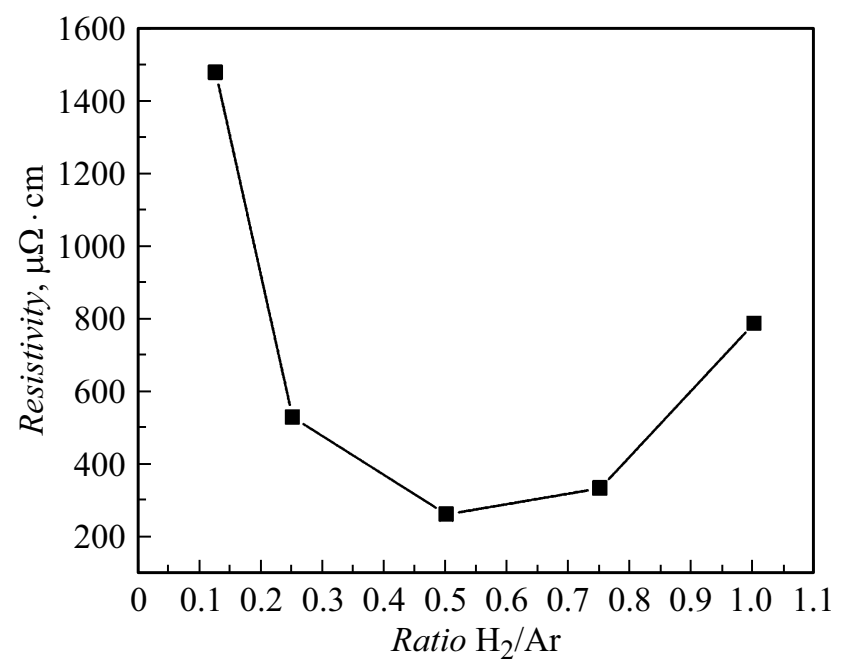

Рис. 1. Зависимость удельного сопротивления пленок нитрида ниобия от отношения газовой смеси реактанта $\mathrm{H}_{2} / \mathrm{Ar}$. 


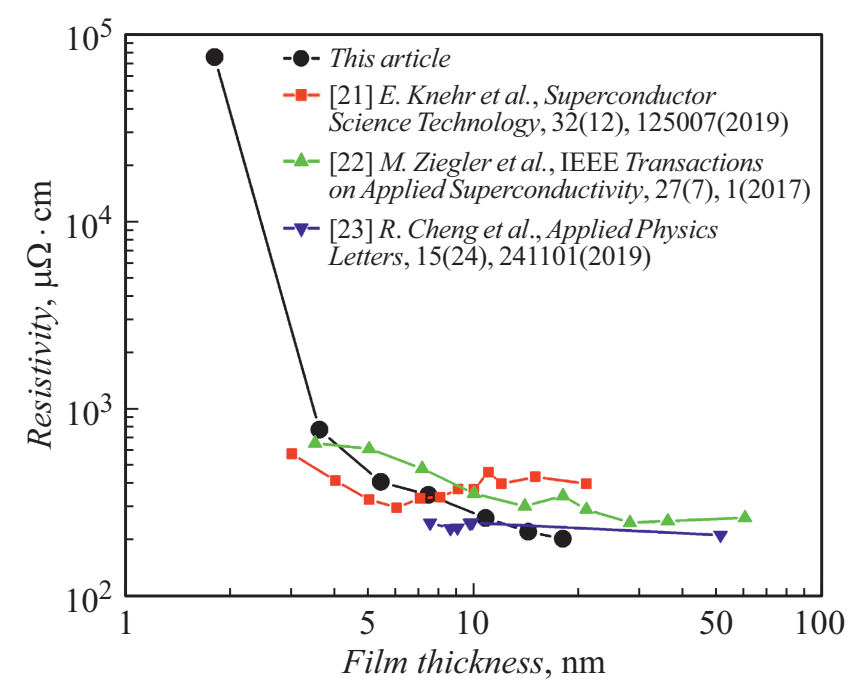

Рис. 2. Зависимость удельного сопротивления от толщины пленки $\mathrm{NbN}_{x}$ для отношения газовой смеси реактанта равным 0.5.

газовой смеси реактанта приводит к росту давления в камере, что в свою очередь ведет к уменьшению потока и энергии ионов, поступающих на подложку. Ввиду этого происходит неэффективное удаление лигандов у молекул прекурсора, которые не прореагировали с поверхностью, что приводит к загрязнению пленки углеродом.

Исходя из величины минимального удельного сопротивления, по которому косвенно можно оценить качество тонкой пленки, режим осаждения с отношением газовой смеси $\mathrm{H}_{2} / \mathrm{Ar}$, равным 0.5, является самым оптимальным. Дальнейшие исследования будут приводиться только для этого режима. Стоит отметить, что полученное удельное сопротивление при оптимальных технологических параметрах близко к значению для объемного нитрида ниобия [24].

Для определения толщины пленки, при которой теряется сплошность покрытия, была изготовлена серия образцов с различной толщиной нитрида ниобия: 1.8, $3.6,5.4,7.2,10.8,14.4$ и $18 \mathrm{~nm}$. На рис. 2 показана зависимость удельного сопротивления от толщины пленки $\mathrm{NbN}_{x}$.

Видно, что зависимость удельного сопротивления пленки зависит от толщины, что говорит о сложной природе роста пленки методом PEALD. Для сравнения полученных результатов в настоящей работе на рис. 2 изображены экспериментальные результаты подобных исследований с использованием различных реактантов [25-27]. При толщине пленки, равной $1.8 \mathrm{~nm}$, удельное сопротивление испытывает резкий скачок, что мы объясняем потерей сплошности пленки. Однако известно, что метод PEALD отличается от других методов осаждения высокой конформностью вплоть до атомарных слоев. Из полученного результата можно судить об островковой природе роста ультратонких металлических слоев методом PEALD.

Измерения на атомно-силовом микроскопе показали, что средняя шероховатость всех образцов с различной толщиной пленки лежит в диапазоне от 2.1 до $2.48 \mathrm{~nm}$. Шероховатость подслоя $\mathrm{SiO}_{x}$ составила $3.4 \mathrm{~nm}$. На рис. 3, $а$ продемонстрирована зависимость средней шероховатости от толщины пленки (выраженной в количестве циклов). Видно, что с увеличением толщины пленки нитрида ниобия шероховатость выходит на плато и определяется средним зерном пленки. На рис. $3, b$ и $c$ изображена морфология поверхности $\mathrm{SiO}_{x}$ и $\mathrm{SiO}_{x} / \mathrm{NbN}_{x}$ $(10.8 \mathrm{~nm})$ соответственно. Из сравнения рис. $3, b$ и $c$ видно, что изначальная шероховатость подслоя $\mathrm{SiO}_{x}$ больше, чем шероховатость поверхности после осаждения пленки $\mathrm{NbN}_{x}$. Можно сказать, что пленка $\mathrm{NbN}_{x}$ планаризует поверхность $\mathrm{SiO}_{x}$.

Для анализа сверхпроводящих параметров получаемых пленок были исследованы критическая плотность тока и температура перехода в сверхпроводящее состояние. На рис. 4 представлен веер кривых зависимости нормированного напряжения (магнитного отклика пленки) от температуры. На вставке изображена зависимость температуры перехода в сверхпроводящее состояние от количества циклов осаждения. Из графиков видно, что пленки, для которых количество циклов осаждения составляло 75 и 100, переходят в сверхпроводящее состояние при температуре 11.2 и $12.4 \mathrm{~K}$, однако имеют высокую остаточную величину магнитного поля, что может говорить об остаточном сопротивлении. Это может означать, что пленка имеет в своем составе большое количество не стехиометрических фаз, которые не являются сверхпроводящими [28]. Авторы предполагают, что химический состав пленки более сложный, а именно представляет твердый раствор $\mathrm{NbCx}$ и $\mathrm{NbN}_{x}$ или сложное нестехиометрическое соединение $\mathrm{NbN}_{x} \mathrm{C}_{y}$. Этот факт подтверждается наличием большого количества углерода, входящего в состав металлоорганического прекурсора TBTDEN. C увеличением количества циклов осаждения и соответственно толщины пленки критическая температура достигает значения $14.8 \mathrm{~K}$ (для 250 циклов осаждения), что является высоким результатом даже для пленок, полученных реактивным магнетронным распылением [29,30]. На ультратонких пленках с толщиной до $\sim 4 \mathrm{~nm}$ сверхпроводящего перехода не наблюдалось вплоть до $4.2 \mathrm{~K}$ (на рисунке не показаны).

Для измерений критического тока на пленках нитрида ниобия $(10.8 \mathrm{~nm})$ формировалась структура типа микромост. Для этого методом лазерной литографии была сформирована тонкая полоска с измерительными контактами. Травление нитрида ниобия осуществлялось в реакторе с индуктивно-связанной плазмой в смеси газов $\mathrm{SF}_{6} / \mathrm{Ar}$ (соотношение расходов 2:1). Снятие резиста осуществлялось в реакторе индуктивно-связанной плазмой в смеси газов $\mathrm{O}_{2} / \mathrm{N}_{2}$. Далее методом взрывной литографии в горячем ацетоне формировались алюминиевые контакты. Характерные размеры структуры 

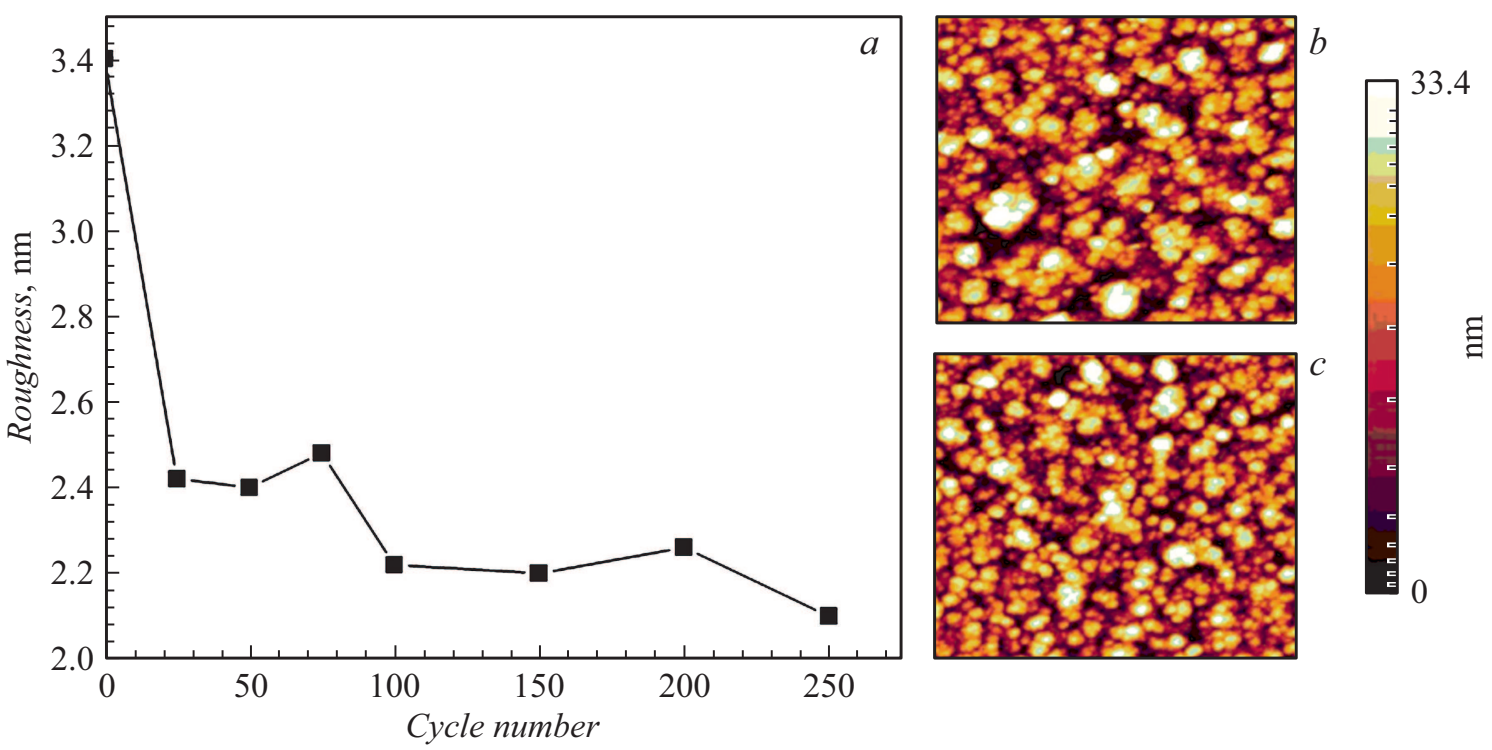

Рис. 3. График зависимости средней шероховатости $\mathrm{NbN}_{x}$ пленки от толщины, представленной в количестве циклов (a). Изображения морфологии поверхности $\mathrm{SiO}_{2}(b)$ и $\mathrm{SiO}_{x} / \mathrm{NbN}_{x}(c)$.

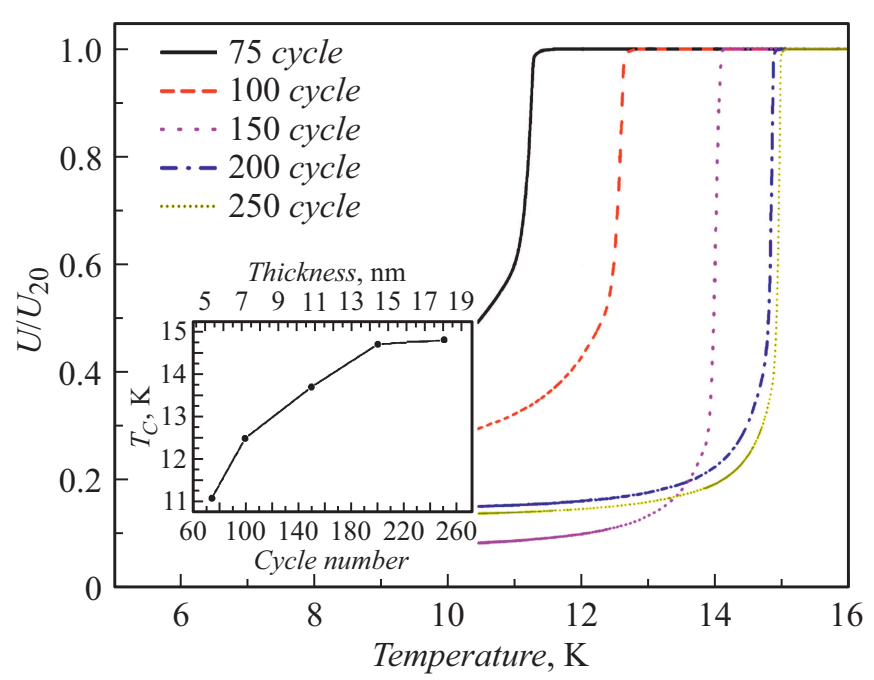

Рис. 4. Веер кривых зависимости нормированного напряжения от температуры для пленок различной толщины (выраженной в циклах). Вставка на рисунке демонстрирует зависимость критической температуры от толщины пленки.

составили $1 \mu \mathrm{m}$ в ширину при длине $60 \mu \mathrm{m}$. Измерения критического тока осуществлялись по двухточечной схеме измерений с использованием малошумящего прецизионного источника-измерителя Keithley 6221A. Охлаждение исследуемых образцов осуществлялось в криостате замкнутого цикла. Измерение и анализ вольтамперных характеристик проводились при температуpe 4.2 K. На рис. 5 изображена типичная вольт-амперная характеристика для сверхпроводящих структур, измеренных в режиме стабилизации напряжения. Данный режим обеспечивает достоверные измерения критиче- ской плотности тока. На вставке изображен внешний вид сформированной структуры.

Величина критического тока составила $83 \mu \mathrm{A}$. Зная геометрические размеры структуры и толщину пленки, рассчитываем критическую плотность тока по формуле: $J_{c}=I_{c} /(d W)$, где $I_{c}-$ критический ток, $d-$ толщина пленки, $W$ - ширина микромоста. Критическая плотность тока для данной структуры составила $0.7 \mathrm{MA} / \mathrm{cm}^{2}$. Известно, что критическая плотность тока для ультратонких пленок нитрида ниобия, осажденных методом реактивного распыления ниобиевой мишени в газовой смеси $\mathrm{Ar} / \mathrm{N}^{2}$, составляет 5-8 MA $/ \mathrm{cm}^{2}$ [31]. В настоящей работе продемонстрирована критическая плотность

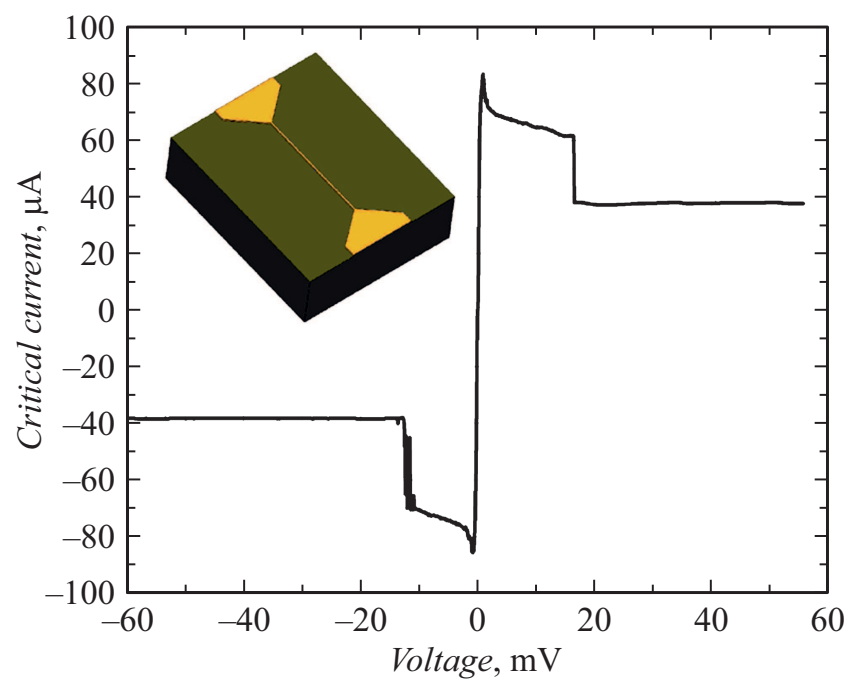

Рис. 5. Вольт-амперная характеристика структуры микромост с шириной $1 \mu \mathrm{m}$ и длиной $60 \mu \mathrm{m}$, измеренная при температуре $T=4.2 \mathrm{~K}$. 
тока на порядок меньше. Для повышения критической плотности тока необходимо усовершенствование состава газовой смеси реактанта.

\section{Вывод}

Пленки нитрида ниобия, полученные методом атомнослоевого осаждения, имеют высокие температуры переходов в сверхпроводящее состояние. Однако пленки имеют низкую критическую плотность тока. Вероятно, одним из факторов, обусловливающих такое низкое значение критической плотности тока, является присутствие углерода в пленке. Присутствие углерода в пленке обусловлено его наличием в самом прекурсоре и неэффективном удалении при росте пленки на шаге воздействия плазмы.

\section{Благодарности}

Авторы благодарят за научную поддержку и плодотворное обсуждение полученных результатов А.П. Сиротину и Е.А. Першину.

\section{Финансирование работы}

Работа выполнена при поддержке проекта № 00042019-0004 Министерства образования и науки РФ. При выполнении работы использовалось оборудование, входящее в УНУ „КУТГИ“ Института нанотехнологий микроэлектроники Российской академии наук.

\section{Конфликт интересов}

Авторы заявляют, что у них нет конфликта интересов.

\section{Список литературы}

[1] A.T. Barton, R. Yue, S. Anwar, H. Zhu, X. Peng, S. McDonnell, N. Lu, R. Addou, L. Colombo, M.J. Kim, R.M. Wallace, C.L. Hinkle. Microelectronic Engineering, 147, 306 (2015). DOI: 10.1016/j.mee.2015.04.105

[2] R.S. Ningthoujam, N.S. Gajbhiye. Progr. Mater. Sci., 70, 50 (2015). DOI: 10.1016/j.pmatsci.2014.11.004

[3] V. Tabakov, A. Chikhranov, Y. Dolzhenko. IOP Conference Series: Materials Science and Engineering. - IOP Publishing, 709|,(3), 033096 (2020). DOI: 10.1088/1757-899X/709/3/033096

[4] G. Ramírez, S.E. Rodil, H. Arzate, S. Muhl, J.J. Olaya. Appl. Surf. Sci., 257 (7), 2555 (2011). DOI: $10.1016 /$ j.apsusc.2010.10.021

[5] C.L. Huang, C.H. Lai, P.H. Tsai, H.A. Huang, J.C. Lin, C. Lee. Electron. Mater. Lett., 9(5), 593 (2013). DOI: $10.1007 / \mathrm{s} 13391-012-2173-0$

[6] J. Hinz, A.J. Bauer, L. Frey. Semicond. Sci. Technol., 25|,(7), 075009 (2010). DOI: 10.1088/0268-1242/25/7/075009
[7] H.X. Lu, L. Kang, J. Chen, Y.Y. Zhong, N. He, Y. Jiang, M. Liang, Q.J. Yao, S.C. Shi. 33rd Intern. Conf. Infrared, Millimeter and Terahertz Waves (Pasadena, CA, USA, 2008), p. 1.

[8] S. Miki, Y. Uzawa, A. Kawakami, Z. Wang. IEEET Transactions Appl. Superconductivity, 11 (1), 175 (2001). DOI: $10.1109 / 77.919313$

[9] W.J. Zhang, L.X. You, H. Li, J. Huang, C.L. Lv, L. Zhang, X.Y. Liu, J.J. Wu, Z. Wang, X.M. Xie. Sci. China Phys., Mechan. Astronomy, 60|,(12), 120314 (2017). DOI: $10.1007 / \mathrm{s} 11433-017-9113-4$

[10] H. Li, H. Wang, L. You, P. Hu, W. Shen, W. Zhang, X. Yang, L. Zhang, H. Zhoi, Z. Wang, X. Xie. Opt. Express, 27 (4), 4727 (2019). DOI: 10.1364/OE.27.004727

[11] B.H. Eom, P.K. Day, H.G. LeDuc, J. Zmuidzinas. Nature Phys., 8 (8), 623 (2012). DOI: 10.1038/nphys 2356

[12] T. Akune, N. Sakamoto, Y. Shibuya. Jpn. J. Appl. Phys., 21 (5R), 772 (1982). DOI: 10.1143/JJAP.21.772

[13] R.E. Treece, J.S. Horwitz, J.H. Claassen, D.B. Chrisey. Appl. Phys. Lett., 65 (22), 2860 (1994). DOI: 10.1063/1.112516

[14] J. Hinz, A.J. Bauer, T. Thiede, R.A. Fischer, L. Frey. Semicond. Sci. Technol., 25 (4), 045009 (2010). DOI: $10.1088 / 0268-1242 / 25 / 4 / 045009$

[15] M. Ukibe, G. Fujii. IEEE Transactions on Appl. Superconductivity, 27 (4), 1 (2017). DOI: 10.1109/TASC.2017.2655719

[16] S. Linzen, M. Ziegler, O.V. Astafiev, M. Schmelz, U. Hübner, M. Diegel, E. Il'ichev, H.-G. Meyer. Supercond. Sci. Technol, 30 (3), (2017). DOI: 10.1088/1361-6668/aa572a

[17] M. Ziegler, L. Fritzsch, J. Day, S. Linzen, S. Anders, J. Toussaint, H.G. Meyer. Supercond. Sci. Technol., 26 (2), 025008 (2012). DOI: 10.1088/0953-2048/26/2/025008

[18] T. Faraz, H.C. Knoops, M.A. Verheijen, C.A. Van Helvoirt, S. Karwal, A. Sharma, V. Beladiya, A. Szeghalmi, D.M. Hausmann, J. Henri, M. Creatore, W.M.M. Kessels. ACS Appl. Mater. Interfaces, 10|,(15), 13158 (2018). DOI: $10.1021 /$ acsami.8b00183

[19] S. Karwal, M.A. Verheijen, B.L. Williams, T. Faraz, W.M.M. Kessels, M. Creatore. J. Mater. Chem. C, 6 (15), 3917 (2018). DOI: 10.1039/C7TC05961B

[20] H.B. Profijt, P. Kudlacek, M.C.M. Van de Sanden, W.M.M. Kessels. J. The Electrochem. Society, 158(4), G88 (2011). DOI: 10.1149/1.3552663

[21] L.E. Archer. PhD thesis (Massachusetts Institute of Technology, Cambridge, 2017).

[22] C.S. Menon, V.S. Pankajaksha. Bulletin Mater. Sci., 3, 187 (1987).

[23] A. Engel, H. Bartolf, A. Schilling, K. Il'in, M. Siegel, A. Semenov, H.W. Hübers. J. Phys.: Conf. Series, 97 (1), 012152 (2008). DOI: 10.1088/1742-6596/97/1/012152

[24] A. Nigro, G. Nobile, M.G. Rubino, R. Vaglio. Phys. Rev. B, 37 (8), 3970 (1988). DOI: 10.1103/PhysRevB.37.3970

[25] E. Knehr, A. Kuzmin, D.Y. Vodolazov, M. Ziegler, S. Doerner, K. Ilin, M. Siegel, R. Stolz, H. Schmidt. Supercond. Sci. Technol., 32 (12), 125007 (2019). DOI: $10.1088 / 1361-6668 / a b 48 d 7$

[26] M. Ziegler, S. Linzen, S. Goerke, U. Brückner, J. Plentz, J. Dellith, A. Himmerlich, M. Himmerlich, U. Hübner, S. Krischok, H.G. Meyer. IEEE Transactions on Appl. Superconductivity, 27 (7), 1 (2017). DOI: 10.1109/TASC.2017.2744326 
[27] R. Cheng, S. Wang, H.X. Tang. Appl. Phys. Lett., 115 (24), 241101 (2019). DOI: 10.1063/1.5131664

[28] M. Chand, G. Saraswat, A. Kamlapure, M. Mondal, S. Kumar, J. Jesudasan, V. Bagwe, L. Benfatto, V. Tripathi, P. Raychaudhuri. Phys. Rev. B, 85 (1), 014508 (2012). DOI: $10.1103 /$ PhysRevB.85.014508

[29] W. Słysz, M. Guziewicz, M. Borysiewicz, J.Z. Domagała, I. Pasternak, K. Hejduk, W. Rzodkiewicz, J. Ratajczak, J. Bar, M. Wȩgrzecki, P. Grabiec, R. Grodecki, I. Wȩgrzecka, R. Sobolewski. Acta Phys. Pol. A, 120, 200 (2011). DOI: $10.12693 /$ APhysPolA.120.200

[30] R. Jha, V.P.S. Awana. Novel Superconducting Mater., 1 (1), 7 (2013). DOI: 10.2478/nsm-2013-0002

[31] I. Milostnaya, A. Korneev, M. Tarkhov, A. Divochiy, O. Minaeva, V. Seleznev, N. Kaurova, B. Voronov, O. Okunev, G. Chulkova, K. Smirnov, G. Gol'tsman. J. Low Temperature Phys., 151 (1-2), 591 (2008).

DOI: $10.1007 / \mathrm{s} 10909-007-9691-4$ 